Article

\title{
Information and Signs: The Language of Images
}

\section{Inna Semetsky}

Faculty of Education and Arts, Research Academic, The University of Newcastle, NSW, Australia; E-Mail: inna.semetsky@newcastle.edu.au

Received: 30 November 2009; in revised form: 25 February 2010 / Accepted: 1 March 2010 /

Published: 12 March 2010

\begin{abstract}
Since time immemorial, philosophers and scientists were searching for a "machine code" of the so-called Mentalese language capable of processing information at the pre-verbal, pre-expressive level. In this paper I suggest that human languages are only secondary to the system of primitive extra-linguistic signs which are hardwired in humans and serve as tools for understanding selves and others; and creating meanings for the multiplicity of experiences. The combinatorial semantics of the Mentalese may find its unorthodox expression in the semiotic system of Tarot images, the latter serving as the "keys" to the encoded proto-mental information. The paper uses some works in systems theory by Erich Jantsch and Erwin Laszlo and relates Tarot images to the archetypes of the field of collective unconscious posited by Carl Jung. Our subconscious beliefs, hopes, fears and desires, of which we may be unaware at the subjective level, do have an objective compositional structure that may be laid down in front of our eyes in the format of pictorial semiotics representing the universe of affects, thoughts, and actions. Constructing imaginative narratives based on the expressive "language" of Tarot images enables us to anticipate possible consequences and consider a range of future options. The thesis advanced in this paper is also supported by the concept of informational universe of contemporary cosmology.
\end{abstract}

Keywords: anticipation; computational universe; ethics; evolution; information; logic of the included middle; memes; Peirce’s semiotics; Tarot images

\section{Introduction}

Since time immemorial, philosophers were searching for a "machine code" of the common language capable of processing information at the pre-verbal, pre-expressive level. Jerry Fodor, a 
philosopher and cognitive scientist, created his Hypothesis of the Language of Thought (LOT) back in 1975. This paper posits human, what are called natural, languages as only secondary to the system of (what philosophers call primitive) signs that are hardwired in humans and which serve as tools for understanding, meaning making and creating new complex concepts by virtue of a code. The paper posits such a system of signs as reflected in, and expressed by, a specific pictorial "language” of Tarot images.

Michael Dummett, famous British philosopher of language and one of the pioneers of the "linguistic turn" in philosophy had a great interest in what he called Tarot history and mystery; he presented Tarot system as a culturally situated card game [1]. This paper's "semiotic turn”, however, does not aim to reclaim Dummett's emphasis on the cultural heritage of Tarot "game”. Rather, its aim is to relatively de-mystify and even naturalize that side of Tarot which is customarily considered to belong to occult science; and to defy in this process the persistent philosophical pessimism expressed yet by Wittgenstein. In the introduction to his Tractatus, Wittgenstein [2] insists that what we cannot talk about we must pass over in silence. The realm that escapes verbal expression is delegated by Wittgenstein to the realm of the mystical even as he acknowledged (in Proposition 6. 522) that things that could not be put into words can still make themselves manifest.

I don't want to argue with Wittgenstein. He was a genius. But I want to demonstrate in this paper that we can indeed express something that appears essentially inexpressible. I want to move away from the limitations of the "linguistic turn" towards what I call the "semiotic turn" in philosophy. By definition, semiotics is the science of signs, linguistic and extra-linguistic alike, and their signification or meaning. According to American pragmatic philosopher and the founder of semiotics Charles Sanders Peirce, philosophy as semiotics acquires a status of proto-science. Analogously, Fodor's [3] LOT hypothesis rests on the naturalistic approach to philosophy as proto-science that presents cognition as computation over compositional mental representations. This means that thoughts are represented in the Mentalese language that allows for complex concepts to be created by combining and re-combining primitive thoughts.

From the semiotic perspective [cf. 4] I consider the organization of Tarot images to be a system of signs the combination of which constitutes the extra-linguistic non-verbal "characters" of the Mentalese "language". The information encoded in the array of pictures may very well be structured in accord with combinatorial (Fodorian) semantics; hence it can be potentially decoded.

Let me first acquaint the readers with some opaque terminology and, in doing so, make it less esoteric and more transparent: What is the Tarot system? It is a deck of 78 pictures where the multiplicity of images may be considered to symbolically represent the archetypes of the collective unconscious posited by Carl Gustav Jung as the memory pool "recording” the collective experiences of humankind across times, places, and cultures. Laurens van der Post, in his introduction to Jung's student Sallie Nichols' book Jung and Tarot: an Archetypal Journey [5], notices her contribution to analytical psychology by virtue of the "profound investigation of Tarot, and her illuminated exegesis of its pattern as an authentic attempt at enlargement of possibilities of human perceptions”.

How can we define a Tarot reading? It is a session between the subject and the reader when the cards are shuffled and then spread in a particular layout. Their meanings are then narrated and interpreted by the reader who "reads" the information from the pictorial "text" that, in a quite astonishing way, becomes available to human consciousness. The reason for such astonishment is our 
deeply ingrained adherence to the direct cause-effect link as a reductive form of mechanistic causality and the absence of the latter in what appears to be just a random distribution of the cards. What takes place, however, is an indirect, mediated, connection akin to the acting principle of synchronicity posited by Jung in collaboration with famous physicist and Nobel laureate Wolfgang Pauli or, in other words, the action of "another kind of causation" [6] and an ensured circular or auto-referential feedback as a feature of self-organization.

Pauli expanded the meaning of the unconscious to accommodate the concept of the field that would have defied the old idea of linear mechanistic causality and expand it to the form of complex “"connections' in nature” [7] so as to be eventually assimilated into natural sciences. Analogously, Laszlo asserted the archetypes' naturalistic status approaching them from a systems science point of view; he argued that they, as well as "the collective unconscious that frames them, are not just "in the mind': they are in nature" [8] embedded in what Erwin Laszlo [9] calls Akashik field that adds the concept of information to the physics of matter and energy. Importantly, the objects of the natural world should be understood as irreducible to solely material things but rather having a proto-mental, according to Alfred North Whitehead's process-philosophy, character.

Contemporary cosmology, too, under the motto "it form bit" and "it from gubit" assigns to the universe a status of a giant quantum computer that processes information in quantum bits called qubits [10]. Information (as envisioned yet by physicist David Bohm) is complementary to both matter and energy and-according to the MIT professor Seth Lloyd [10]-it is on the basis of this information that the universe computes its own dynamical evolution and in this process it is capable of actualizing potential reality as the computation proceeds. Ditto for the evolution of the human mind and the whole of culture: mind and intelligence are evolving. Intelligence is an eventual outcome of the evolutionary and learning process called by Charles Sanders Peirce semiosis.

The computational approach needs a bit (no pun intended) of qualification. At the cutting edge of philosophy of mind and cognitive science computers are understood as dynamical systems that indeed manipulate "bits", but these units of information are not strictly reducible to what in physics would be called particles. They are moments in the flow represented-importantly-by analog, and not solely digital, information. Therefore they can be defined as discrete "bits" only within a certain context, that is, always taken as parts-of-the-whole [cf. 11]. Lloyd [10], stressing that quantum (universal) computation, sure enough, proceeds in a dual analog-digital mode, specifies the structure of the computational space in terms of a circuit diagram representing both logical gates (the places where qubits interact thus exchanging/transforming information) and, importantly, causal connections represented by the connecting "wires" or paths along which the information flows.

The very meaning of information as a measure of communication suggests "a channel which can carry a unit of communication with the least amount of uncertainty" [Prigogine in 8]. Tarot system, functioning as an unorthodox channel, transforms or redistributes information by conveying messages therefore contributing in some degree to decreasing epistemic uncertainty. The computational space is expressible in multidimensional geometry which models knowledge that apparently "we know but cannot tell” [11]. Still, even as we appear ignorant, we become capable of "telling" the otherwise untold story by means of Tarot readings and the interpretation of images and pictures that serve as a material carrier for messages. It is "the constitution of messages [that] forms the subject matter of semiotics” [12]. What takes place is the construction of imaginative narratives using Tarot symbols as 
non-verbal signs to be narrated and interpreted. Imaginative narrative is but one of the methodologies of the interdisciplinary field of Future Studies.

Because universal experiences across cultures, times, places and spaces are "contained" within the symbolic level of the collective unconscious, the semiotic interpretation of the deep meanings encoded in the Tarot pictorial language contribute to our learning from this very experience, thus to our growth and the evolution of consciousness in both intellectual and moral terms. The human mind evolves by means of an expanded epistemic access to the level which would have otherwise remained beyond reach [13]. This level has acquired the name semiosphere given to it by the Russian semiotician of the famous Tartu school Yuri Lotman [14].

Lotman's term has undergone its second birth when recently posited by molecular biologist Jasper Hoffmeyer who defined semiosphere as a holistic structure that "penetrates to every corner of these other spheres [the atmosphere, the hydrosphere, and biosphere], incorporating all forms of communication [and constituting] a world of signification” [15]. Contemporary semiotician John Deely suggests the all-encompassing term signosphere to pay tribute to what he calls Charles Sanders Peirce's grand vision that has the advantage of being rooted in science rather than in mysticism [16].

Back in 1975, general systems theorist Erich Jantsch included Tarot in his systematic overview of approaches and techniques of what he called the "inner way" to knowledge, placing Tarot at the mythological level among genealogical approaches, yet acknowledging the relation of such a mythological level to the level identified as evolutionary. Jantsch claimed that it is at this particular level where the human mind becomes potentially capable of "tuning in ... to the evolutionary wave-form [and] developing a consciousness capable of relating to a four-dimensional reality” [17]. Pointing out that the organization of systems proceeds through self-realizing and self-balancing processes, Jantsch prophetically suggested that "Tarot cards... may be embodying [and] mapping out the field of potential human response".

The 78 pictorial cards are traditionally called Arcana. The meaning of the word Arcana (or Arcanum, singular) is that what is necessary to know, to discover, to anticipate, so as to be fruitful and creative in one's possible endeavors. Arcana derives from Latin arca as a chest; arcere as a verb means to shut or to close; symbolically, Arcanum is a tightly-shut treasure chest holding a secret: its implicit meaning. Arcana are akin to the infamous universals of communication that however exceed their linguistic representations: they are extra-or trans-linguistic and "located' at the level of semiotic, pre-conscious and non-verbal, signs. The symbolic messages encoded in those signs express themselves by virtue of "speaking" in the Mentalese code.

If and when decoded-that is, made available to consciousness-they become a powerful motivational force to facilitate our actions across the emotional, cognitive, and/or behavioral levels and thus to accomplish important cognitive and ethical objectives, especially considering that we live at the time of global conflicts, religious misunderstandings, and political bifurcations when the problematic of shared meanings and mutual understanding is of paramount importance.

A Tarot reader is a trained practitioner who has developed her intuition so as to secure readings of a high reliability. An expert reader, like myself, is also a qualified professional that gained her skills through a life-long education, personal development and professional training. Tarot readings-as the interpretation of symbols constituting a specific layout or spread-bring unconscious implicit contents to the level of explicit conscious awareness. According to Nobel Prize winner Herbert A. Simon, 
"a symbol is simply the pattern, made of any substance whatsoever that is used to denote, or point to, some other symbol, or object or relation between objects. The thing it points to is called its meaning" [18], and the ability to decode the images so as to "compute" their deeper symbolic meanings is a prerogative of a Tarot reader.

As Simon [18] points out, "computers were originally invented to process patterns denoting numbers, but they are not limited to that use. The patterns stored in them can denote numbers, or words, or lizards, or thunderstorms, or the idea of justice. If you open a computer and look inside, you will not find numbers (or bits, for that matter); you will find patterns of electromagnetism". In our search for the Mentalese we may indeed wish to "open” the human mind and probe inside it, but we won't find anything in this intangible medium. Still we may find something if we consider the mind as projected though quite tangible properties of the pictorial cards with their picturesque images that carry powerful symbolic meanings. From the perspective of projective hypothesis that in its various guises plays an important role in the area of psychological testing and assessment, the constellation of Tarot pictures presents itself as an expanded scope of space and time accessible to observations [cf. 19]: because of projection, the invisible realm of the collective unconscious (what Plato would have called the realm of the intelligible) is spatialized and rendered visible (what Plato would have called the realm of the sensible).

In contemporary cosmology the suppression of many dimensions (which happens for example when a $3 \mathrm{D}$ object is projected on a $2 \mathrm{D}$ surface not unlike cinema projection) is called compactification. Lloyd [10] points out that "most information is invisible" and it takes energy to process information, to make it relatively visible at the level of matter, that is, to compactify it. The basic material elements such as "Earth, air, fire, and water...are all made of energy, but the different forms they take are determined by information. To do anything requires energy. To specify what is done requires information. Energy and information are by nature (no pun intended) intertwined" (brackets in original). Therefore we may consider matter, energy and information "intertwined" in a self-referential, triadic, relation as shown on Figure 1:

Figure 1. A triadic relation.

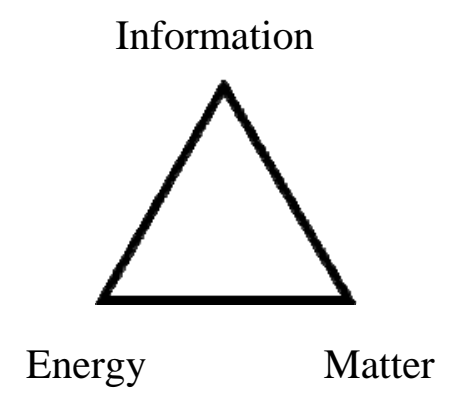

According to Charles S. Peirce, it is the necessarily triadic structure that constitutes a genuine sign. We will explore such similar triadic, self-referential, structures further below as the paper proceeds. It is the self-reference of a system that makes it, in a way, self-transcending or evolving. As recently noted by Kelso and Engstrøm in their book "The Complementary Nature”, "sentience and self-reference have been making trouble for philosophers for centuries” [20]. Kelso and Engstrøm use a squiggle, tilde “ ”, as a symbol for pinpointing the relation between what otherwise would have been 
considered dual opposites and assert that in "the case of human beings, complex nonlinear self-organizing [self-referential] systems of energy matter have managed to evolve to the point of organizing a sense of self other" (brackets mine).

Different "self other" (self $\sim$ not-self) pairs do belong to a variety of discourses; their commonality derived from the same relational dynamics “contained” in the logic of the included middle (tilde “ ”). Physicist and philosopher Basarab Nicolescu [21] also refers to the logic of the included middle in his contrasting in vitro (disciplinary) with in vivo (transdisciplinary) knowledge. Disciplinary knowledge is based on the classical logic of the excluded middle that induces a separation between subject and object and reduces the meaning of knowledge to knowing merely the "objective" facts of the external world. The new transdisciplinary knowledge however is founded on the logic of the included middle that connects subject and object so that they, in Nicolescu's words, correspond to each other. They are in a triadic vs. dyadic relation.

Nicolescu comments that personal growth inevitably passes through a trans-personal dimension, when "self", in an anti-dual manner, can become "other". The emphasis on correspondence or communication indicates that there is an interdependent network in which each level "speaks" to each other, desperately trying to understand each other's expressive "language", to thus create shared meanings along the communicative link expressed by “ ”. Such unorthodox logic is akin to what contemporary mathematician Louis Kauffman calls virtual, or archaic, logic that "goes beyond reason into a world of beauty, communication and possibility" [22] as well as beyond given facts into a world of interpretable symbols, meanings and values. The apparent dichotomies and antinomies of "either/or" habitual thinking are being transcended and traversed by virtue of the "both-and" that constitutes a self-referential relation as a basis for the science of coordination dynamics equally applicable to natural and socio-cultural systems.

As stated by Daniel Dennett [23] with regard to the problem of consciousness, computational structures are just that, self-referential, that is, capable of self-understanding. In other words, and contrary to Cartesian dualism, the supposedly detached observer becomes what physicist Henry Stapp has recently called a "participating observer" [24]; and subject and object reciprocally presuppose each other. It is the structural self-reference that generates a string of (seemingly meaningless) signs that nonetheless acquire meanings when positioned in specific and, importantly, evolving contexts. Because of self-reference, the subject of the Tarot reading becomes capable of self-transcendence: she becomes aware of her own dynamical evolution by means of forming her "own world of symbolic representation of reality" [19] embodied in the array of pictures.

Self-transcendence has been defined by Erich Jantsch as "the creative overcoming of the status quo" [25]. The general paradigm of self-organization is not limited to material structures only: it "embraces...also mental structures, such as ideas, concepts or visions" [19]; in other words, the world inhabited by signs as archetypal patterns that are both sources and destinations of information. Jantsch's prophetic mind envisaged that "the self-organization of information is an aspect of the self-organization of life and the gestalts it produces are the gestalts of life. They are autonomous as are the gestalts of other autopoetic system dynamics. They....are capable of emancipating themselves from reality. Thus they can change and redesign reality" because they can anticipate "the options in [their own] further evolution". In the self-organizing process "characterizing the system and its 
relationship with the environment ...mind... is no longer the opposite of matter, but...co-ordinates the space-rime structure of matter”.

Pictures, it is said, are worth more than many thousands of words and can be used so as to make implicit or explicit inference to derive a meaning for the image. Especially if they denote (as Simon points out) the idea of justice-and it is precisely this idea of Justice that happens to be the major card number XI carrying this name; or Strength-the card number VIII; or Temperance-the card number XIV; and so on. Simon described such an inference in terms of the recognition of features that would have given an experienced person some reliable cues of how to interpret it and suggested that specific “recognition capabilities account for experts' abilities to respond to many situations 'intuitively'... [and not] to hypothesize additional mechanism to explain intuition or insight” [18]. I disagree with Simon on this point and have earlier explained the functioning of intuition in terms of Peirce's logical category of abduction as an implicit and tacit, bordering on infinitesimal, hypothetical inference [see e.g., 26].

\section{Discussion}

When combined in a layout, Tarot cards form a visual semiotic system that may be "read" like any other text, that is, its patterns recognized providing of course that both syntactic and semantic rules that parallel some formal code as the expressive meta-language comprising the "keys" to Tarot symbolism, is known to an expert reader who is able to decode them, that is, to translate them into a spoken language. This is the so-called Celtic Cross spread (Figure 2):

Figure 2. The Celtic Cross spread.

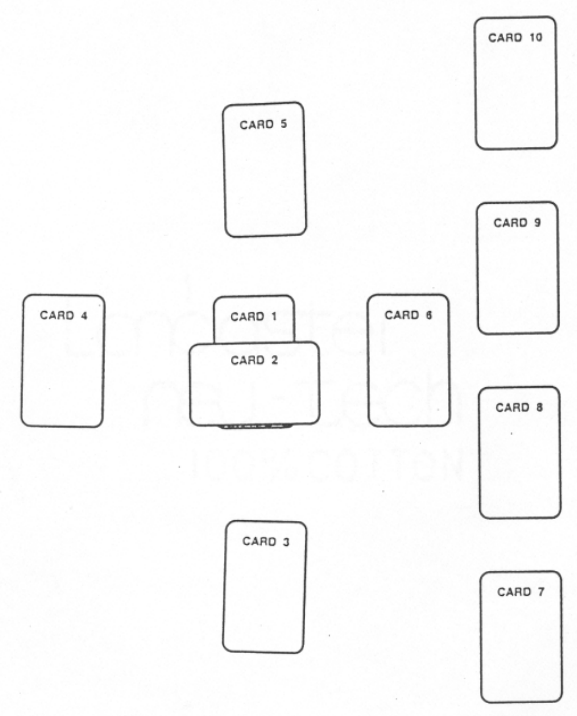

This layout comprises ten positions, signification of which may be considered to carry specific connotations, but it is not exhausted by them. Their meanings are codified; however-and importantly-codification is never fixed but itself represents a dynamic evolving process that includes a pragmatic dimension: it is "usage [that] renders the sign more precise and extends its convention" [27]. 
This means that as a result of the interplay of nature and culture, codification (a presupposed rule, law etc.) is evolving and is subject to dual mode, analog and digital.

Such dual codification pertains also to what Daniel Dennett called memosphere [28]. Not aware (apparently) of Yuri Lotman's earlier approach to the semiotics of culture, Dennett coined memosphere as a concept that appears to be remarkably similar to semiosphere. Dennett posits a naturalistic framework for human experience and ethics with regard to the function of memes as complex ideas or habitual patterns that replicate themselves as they pass on via culture and communication in human actions and history, hence enabling evolution and cultural growth. In a manner analogous to the relatively autonomous Jungian archetypes, memes are considered by Dennett to be potentially immortal entities that literally enter into human minds while functioning quite independently of one's conscious will or volitional choices. Incidentally or not, the theory of memes sometimes invites the same scepticism as, historically, archetypes or Tarots; up to the point of taking a "meme theory...not much more valuable that any astrological or otherwise esoteric interpretation of the meaning of the universe and its inhabitants" [29]. In fact, memes often circumvent our explicit choices quite in accord with Jung's notion that archetypes can easily "possess” individual or collective (cultural) psyche. Because of the archetypal, that is, bordering on possessive and forceful, nature of memes, they may "spread...within a group...without individuals making explicit, reflective value judgments” [30]. Becoming conscious of the unconscious action of the memes (or the archetypes) constitutes the process called managing the memes, and the physical implementation of the archetypes in Tarot pictures supplies an actual body to an otherwise virtual (non-physical) "meme machine”.

Tarot archetypal images therefore are what in today's parlance would be called a machine meta-language "describing” the evolutionary process that consists of the taking of habits (as Peirce would have said) comprising our memes as canonical codes [cf. 31]. As noticed by Markoš [31] with regard to semiosphere, it is a specific meta-language that ensures a relative identity of codes when it performs a function of transmitting a message. In this sense, a constellation of images representing the archetypes of collective unconscious cannot be taken just as random parochial combination.

Providing that a semiotic code serves as "the correlation or correspondence between sign repertoires ... and their meanings" [32], each position as per Figure 2 may be considered to have the following connotations [cf. 33] :

Position 1. The subject's presenting problem, or an area of a particular concern to the subject of the reading.

Position 2. The influence, such as impulses, feelings, traits, or behavioural patterns (not necessarily the subject's own), or some other sign that may strengthen or weaken the problem the subject is concerned with, as per position 1. Quite often, this position signifies some, as yet unperceived, obstacles.

Position 3. Some past unconscious factors that contributed to the present situation. The "roots" of the matter in question which are deeply embedded in the unconscious and may appear, quite often, in the subject's dreams.

Position 4. A significant moment in the subject's past history that still affects the situation and whose implications are so strong that they might show up in the subject's 
future dynamics. Even if the subject did not pay particular attention to it and almost "forgot" it, such a memory, if significant, comes out in a reading.

Position 5. A potential, or coming into being, future. Perhaps some motivations, even if outside of the subject's conscious intent, have contributed to this development, which thereby shows it presence, even if only as a trace of "the memory of the future".

Position 6. The further development of the situation as it unfolds in the immediate future.

Position 7. The subject's current state of mind comprising thoughts, accompanied by affects, shows up in this position. The subject's own perceptions may be quite overwhelming to him/her, or even obsessional.

Position 8. The subject's immediate environment, that is, home, support system (or the lack thereof), family, friends, partners, relatives, business associates, in short people representing significant others for the subject in relation to his/her presenting problem.

Position 9. The subject's hopes and wishes, aspirations and ideals, are shown here. They are often accompanied by fears or anxiety.

Position 10. A possible outcome of the current dynamics as it envelops all contributing and hindering factors represented by cards that will have occupied each position.

We can see the past-present-and-a-possible-future distributed in the same layout of pictures and enabling us to anticipate what may appear ahead. Jantsch [19], from his systems-theoretical perspective, acknowledged that any self-referential (self-organized) system has a feature of anticipation: the system's present state contains "not only the experience of past evolution, but also the experience of anticipated future [that] vibrates in the present”.

All positions provide a rich context within which the images of particular cards are to be interpreted. It is by now obvious that some positions in the spread do correspond to what philosophy of mind describes as propositional attitudes and that encompass such common semantic categories as beliefs, fears, desires, and hopes. Even when being "located" at the unconscious sub-personal level from the subjective point of view, our beliefs and desires objectively do have a rational compositional structure quite in agreement with Fodor's LOT hypothesis. This structure can be laid down in front of our own eyes in the format of Tarot pictures as physical implementations for mental representations for affects, thoughts, and actions alike.

Depending on which particular card will "fall out" into a specific position as described above, an expert reader can interpret or read signs in accordance with the specific semantic context. But also applying in practice or decoding unorthodox grammar, or syntax as based on the logic of the included middle constituting the core of semiotic analysis: that is, from "the observable signifier, we infer by mediation of the signified in a process of drawing a logical conclusion about what the sign stands for" [32]. And the images on Tarot signs stand for real experiences embedded in the collective unconscious. Significantly, we have to use a "key" to "crack" the Mentalese code notwithstanding the fact that such mediation is not fully explicit but tacit and intuitive.

Even if such step-by-step computational rules cannot be articulated fully in a human language prior to its very interpretation; still, as dynamical patterns, they are mimicked in human behaviors. Memes do manage and re-enact themselves in such a way that this enactment may very well be called an “intelligent but unthinking behavior” [28]; such objective intelligence (objective psyche, as Jung called it) 
always already preserved in the form of the unseen, yet potentially active and formative, information. According to Lloyd, the universal laws "preserve information as it is transformed" [11].

The process of managing the memes at the conscious level, however, becomes possible under the condition of transforming the invisible information into visible when we become capable of decoding the language "written” in Mentalese characters and comprising the unfolding "story" projected in the array of pictures. With the help of imagination, insight, and abduction-an unorthodox mode of inference which nonetheless has a power "of guessing right" [6] even while being pre-conscious and not rationally controllable-we articulate the pictorial text constructing in this process an imaginative narrative for the past-present-and would-have-been-future experiences. Sure enough, the boundary line separating the unseen invisible information from the visible is quite subjective and depends on our own ignorance vs. knowledge: Seth Lloyd is adamant that the invisible information-entropy-is also the measure of ignorance.

The task of making the unconscious conscious (making the invisible present) is accomplished by us understanding the Mentalese (that is, ourselves becoming parts-of-the-whole hence capable of anticipating some parts of the whole) encoded in the structure of that meta-machine which is Tarot semiotic system.

Tarot functions in the two-fold manner reminiscent of the second-order cybernetics [cf. 34,35]: both as an amplifier by rendering the subtle aspects of one's psyche vivid and substantial, and as a positive feedback that directs the amplified (made manifest or visible) information back into an expanded system, thus equipping it with information (neg-entropy!) by having made the latent unconscious contents manifest and rendering them meaningful.

The process of reading is itself a peak of fluctuation in the open-ended interaction: the content of one's mind is as if "estranged" for a moment, creating the instability phase between the subject of the reading and the current level of his/her knowledge (or, rather, ignorance as Lloyd would say), this phase "in which novelty breaks in, the law of large numbers is rendered invalid and the fluctuations of consciousness prepare the decisions for the next autopoetic [self-referential] structure" [19] (brackets mine).

An invisible realm acquires visibility and legibility, and in this respect the pictorial text of a Tarot layout is a result of the self-organization in action [cf. 10]. Sure enough, information albeit conserved-or preserved, as Lloyd says-is being redistributed, contributing to a new "construct” having appeared at a higher level of organization. In this sense, active (creative) interpretation as the included middle between oneself and the world creates the "self other" complementary pair that exceeds just a passive adaptation to one's environment: evolution should be understood as a reciprocal co-evolution which is only natural because in the computational universe biology is complementary to physics.

Renee Thom, a founder of the catastrophe theory in mathematics, acknowledges the necessary isomorphism of forms and makes it clear that the correspondence is produced by interaction or “coupling”. In the case of projected shadow, for example (and as we said earlier both projection and compactification are important for understanding the Tarot structure and function) isomorphism is maintained because the light, illuminating the original and casting the shadow, performs the function of interaction (we may say, of the included middle). Thom notices that stability of biological forms demands them having a dynamically physical character, that is, they depend on constraints imposed by the physical level. 
In this way, "the organic release of evolution allows the appearance of forms, more refined, more subtle, more global...and...charged with meaning” [36] or implicit signification as a manifestation of the universal irreversible dynamics. The archetypal forms would have been located at this global symbolic (semiospheric? memospheric?) level. Thus we cannot avoid but accept the mediation of biology and physics by culture and human experience; and the universal experiences (notably both actual and potential) are inscribed in the images of Tarot cards and are encoded in the language of symbols.

Robert Rosen, a father of mathematical biology, also talked about the universe as seemingly embodying the semantic, akin to the natural language, dimension. Yet, while focusing on biology and "life itself" [37] he remained agnostic on the problem of effective calculability unless we take it for granted that Nature itself (with its laws of physics) "speaks" a language of sorts. Rosen quotes from Martin Davis' earlier book (which precedes Seth Lloyd's research by nearly a half of the century) Computability and Unsolvability (1958; New York: McGraw-Hill): "For how can we ever exclude the possibility of being presented someday (perhaps by some extraterrestrial visitors,) with a (perhaps extremely complex) device or 'oracle' that 'computes' a noncomputable function? (p. 11)”.

This question becomes moot in the framework of the present paper: such an "oracle" exists and the material in this paper demonstrates that it in a certain sense does compute the (otherwise) incomputable. I remind the readers about Dennett's insistence that computational functions are self-referential, and one example of such a self-referential structure is presented in a semiotic triangle on Figure 1, indeed. The triadic structure reciprocally unites matter, energy and information in the universe which functions as a quantum computer that computes its own dynamical evolution. It is the logic of the included middle that makes Peircean triadic sign (Figure 3) functional.

The triadic sign is a sign which is potentially evolving towards higher levels: for Peirce, signs grow and become other, more developed or evolved, signs. The whole triad of sign-object-interpretant becomes an object for the next sign that will have needed further interpretation according to the dotted line as shown on Figure 3:

Figure 3. Peirce’s triadic sign.

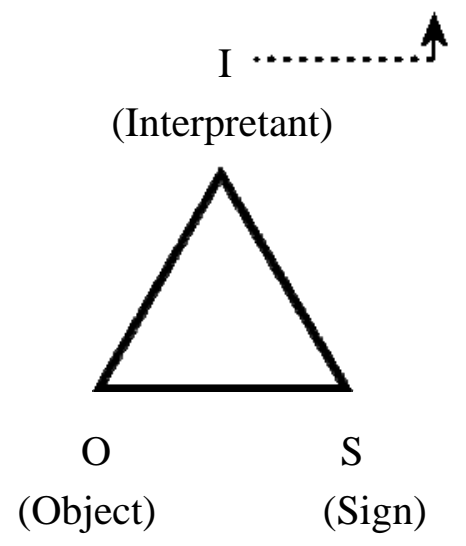

As a semiotic system, the symbolic level of memes or archetypes encoded in proto-Mentalese (that notably "belongs" not to an individual Cogito forever separated from the world, but to the objective psyche of the Jungian collective unconscious) creates a semiotic bridge between the worlds of mind 
and matter by establishing a sort of interobjective (using Bruno Latour's term) relation. Contemporary post-Jungians view Jung himself as a systems-theorist, and "a systemic...view implies that...inner and outer...interpersonal and intrapsychic can be seen to be [a] seamless field of references” [38] that unite in a holistic manner the otherwise binary opposites of mind and matter, the knower and the known.

In one of his letters to Jung, Pauli expressed his belief in the gradual discovery of a new, what he called "neutral”, language that functions symbolically to describe an invisible, potential reality. This level of semiotic reality, while not being observable by senses, is nonetheless inferable indirectly-via mediation-through its observable effects. Such unorthodox language represents the means of communication crossing over the psycho-physical dualism [39].

Why neutrality of such a language envisaged by Pauli back in 1952? As Lloyd [10] tells us, neutrons are capable of "saying” both yes and no simultaneously; apparently they participate, as Kelso and Engstrøm would have said, in the "yes no" triadic relation. The invisible information (entropy) is a measure of ignorance, and by making the invisible visible it may seem that the entropy will decrease but in fact the total amount of information is preserved-it is just redistributed because of the shifting boundary between (the classical) observer and the observed when the interpretation of symbols (the included third of the Peircean interpretant) enables the evolution of consciousness.

So Tarot symbolic language, which-like any language in general-is represented by a structured system whose role is to pair expressions (functioning as public aspects, e.g., physical marks) and messages (private aspects: thoughts and concepts), that is, to have the means for mapping between them [cf. 40], performs a function of the interpretant that conforms to the self-referential logic as semiotics, that is, logic of the included third, the included middle; a paradoxical illogical or "virtual" logic described by the mathematics of fixed points [20,22].

The images render themselves interpretable, and interpretation itself is based on the analogical reasoning so that meanings-at the level of their expression in verbal language-appear to as if created anew. This apparent creation ex nihilo is not an occult or mystical feature: Lloyd explicitly emphasizes that "quantum mechanics, unlike classical mechanics, can create information out of nothing" [10] (italics in original). The parts of the universe that as a whole functions as a quantum computer are entangled (always already parts-of-the-whole), and it is this entanglement that allows for the genesis of information despite the apparent “spookiness” of Einstein's old action at a distance.

In the evolution along the stages represented by major Arcana from Zero (the card called "The Fool") to the card numbered XXI ("The World") the limited world available to sense-perception expands. Each image may be interpreted at the psychological, epistemic or ontological levels. The first card is called The Fool (Figure 4) and is traditionally unnumbered, that is, it has Zero as a signifier.

Zero numbering is extremely significant. Recall Lloyd's argument that in the computational universe information can be created seemingly out of nothing, that is, out of the state with zero entropy. In philosophical terms, it is akin to the old and seemingly unsolved problem of being as first known, ens primum cognitum [42]. This is the state of total freedom (total Chaos) and unbounded, even if yet invisible, information. The potential for information-processing is at its maximum, and the Fool will start on its experiential journey of discovery, computing itself and evolving towards higher levels of complexity.

As asserted by Lloyd, the computational universe (contrary to the old mechanistic paradigm) gives rise to the possible forms of order due to its "innate information-processing power" [10] and because 
of the structural dynamics of the earlier mentioned logic gates whose action "completely determines what happens during the computation”. By symbolically performing a quantum leap into the abyss just in front of her (Fig 4), the Fool performs a quantum of action and thus begins the evolutionary process.

Figure 4. The image of “The Fool” [41].

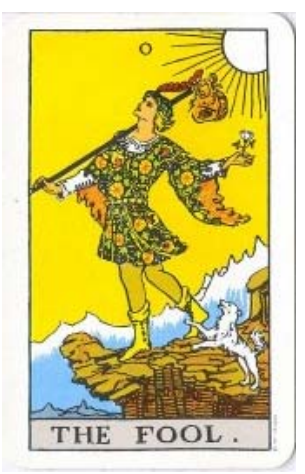

\section{Result}

At the level of our real experiences, we say that we step in the experiential life-world and, by means of the information-processing, begin to understand the meanings of this and subsequent experiences: we become wiser and our consciousness expands to accommodate and realize that which presented itself as just invisible information. Citing Lloyd again, "it is nothing wrong with beginning from nothing. For example, the positive numbers begin from zero (the 'empty thing')”. Indeed, the Fool is followed by the picture indicated by the Roman numeral I and called “The Magician” [43] (Figure 5):

Figure 5. The image of “The Magician”.

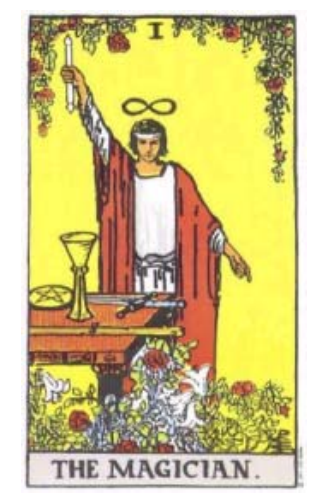

From the human perspective, the creative act of enhanced perception as represented by "The Magician” is necessary for the re-organization of experience by making it meaningful, that is, producing order out of initial perceptual (or ontological) chaos so as to understand its implicate yet logical (semiotic) structure, to make sense out of it. A creative (abductive) act precedes the drawing of dyadic propositional conclusions because human experience and the whole of culture, including past heritage and future possibilities embedded in the collective unconscious act as the included third between what otherwise appears to be the two disparate Cartesian substances, mind and matter.

According to G. Spencer-Brown's Laws of Form, logic is being constructed on the basis of an initial act of making a distinction marked in a series of the multiple bracketing $\{\ldots\{\ldots\} \ldots\}$ that repeat 
or replicate themselves. The information (which is always already preserved even in its otherwise imperceptible potential form) becomes visible and useful. The process is analogous to the number series represented by numbers that start from 0 (as the empty set $\varnothing$, an abstract entity of mathematical analysis akin to the zero numbering of The Fool), to the whole numbers series from 1 to 21 that correspond to the sequence of the Major Arcana. Each subsequent picture (with a corresponding number) reflects a particular pattern of thought, mood, affect etc.; each pattern may be represented by a set with a certain number of elements that re-iterate; hence, generate themselves [cf. 44]. Such evolution is illustrated by Figure 6:

Figure 6. Infinite series [from 45] [cf. 46].

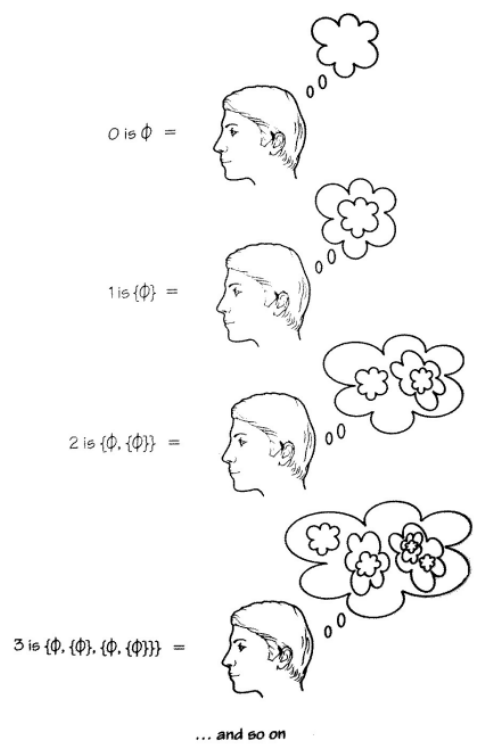

Even as numbers proceed in their natural progression, the human psyche functioning as "unextended intensity" [Jung in 8] is typically marked by tensions or bifurcations, which signify "a fundamental characteristic in the behavior of complex systems when exposed to high constraint and stress” [47]. During readings, a Tarot layout may indicate the presence of a highly unstable situation or a state of mind; even if the mind itself, at the conscious level, may be quite unaware of its own situation it still can feel or anticipate the latter's emotional impact or be in a certain affective state.

The outcomes of such a tension imposed on a system will vary: similar to the bifurcations classified according to their degree of manifestation, as well as the dynamic regime in which a system will potentially settle, various major cards are signs of either subtle (e.g., "The Wheel of Fortune”), catastrophic (e.g., “The Death”), or even explosive (e.g., “The Tower” [see 48]) bifurcations.

"The Tower" (Figure 7) is a symbol of total destruction: 
Figure 7. The image of “The Tower”.

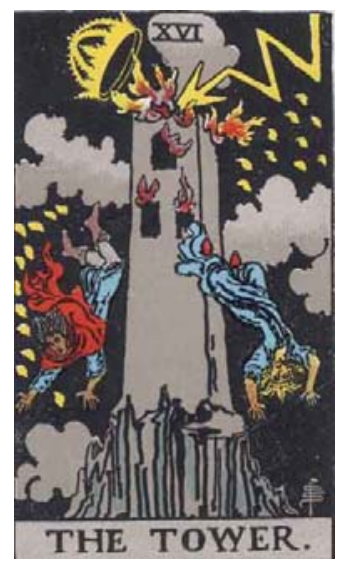

At the level of socio-cultural reality, this image has an uncanny resemblance with the image of the destroyed Twin Towers on 9/11 as shown in the photo below (Figure 8) found on the Internet:

Figure 8. The photo of the Twin Towers on 9/11.

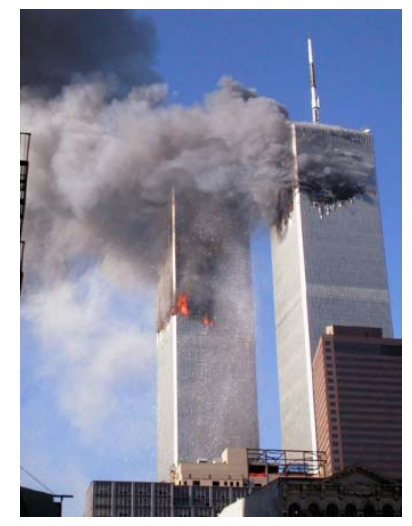

At the deeper level, its symbolic meaning is that of the Tower of Babel; in fact, it is portrayed in this manner in some decks, like in The Lovers' Tarot (Figure 9) that incorporates the elements of the famous painting.

“The Tower" card is a symbol of false omnipotence and mistaken certainty, a priori condemned to destruction during the most powerful and confusing instance of the collision of opposites and amidst persistent contradiction and mutual misunderstanding: the confusion of tongues, indeed. The ultimate destruction-a body turned into a life-less skeleton-is seen in this other poignant and maximally real image of 9/11 also published on the Internet (Figure 10).

Thunder and lightning as per the image of "the Tower" are the universal signs of the wrath of gods; the symbolism of which also indicates a swift-and painful-alteration at the level of collective consciousness when it observes the aftermath of the destruction of the self-erected unstable structure. Significantly, “The Tower” is immediately followed by the picture called “The Star” (Figure 11).

The presence of "The Star" in a deck, as a natural progression from "The Tower", is a symbolic message that no destruction is final. Evolution has a direction and is presently (following the disastrous start of the 21st century symbolized by “The Tower”) oriented towards “The Star”. Narrating the 
image brings forward the meaning of hope, healing, inspiration and the new Aquarian age symbolized by the naked woman pouring waters; in fact, this card is sometimes called The Star of Hope.

Figure 9. The image of “The Tower” [49].

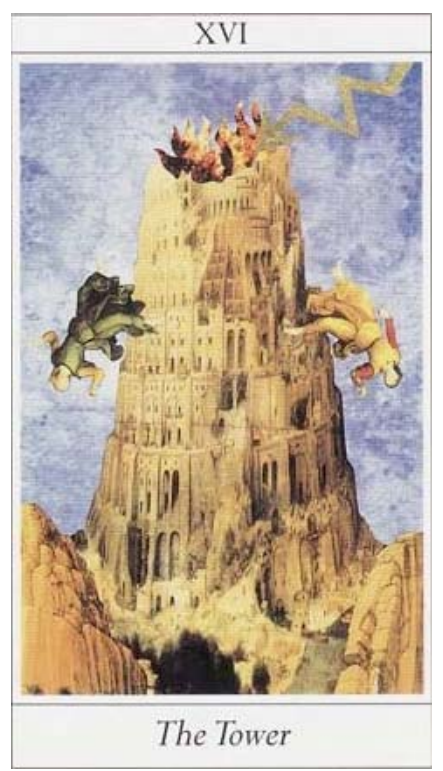

Figure 10. The photo of the destroyed Twin Towers.

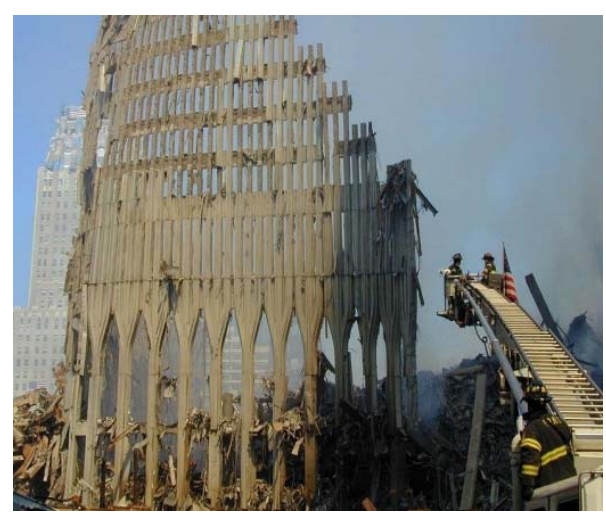

Figure 11. The image of “The Star”.

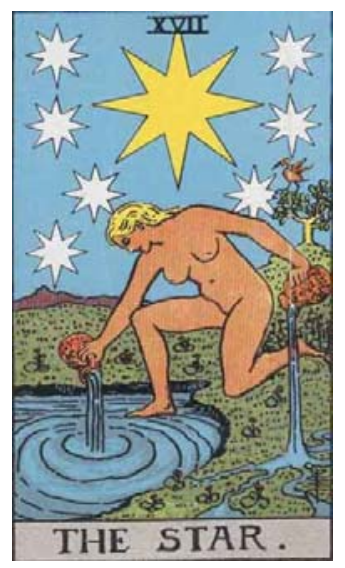


In the current global climate permeated by diverse beliefs, disparate values and cultural conflicts when different memes "compete", as Dennett would have said, with each other at the global level and have led to destructions of "The Tower" scope, the universal value of Hope is paramount [see 48]. Significantly, we can make it our reality if we step into the informational process of our own evolution so as to become able both to understand and transform it by virtue of becoming able to anticipate possible consequences of our own actions. Tarot readings provide the means that enable proper self-reflection so that we can begin to learn "how to do the right thing at the right time" [11] choosing ethical actions in harmony with the universe.

The figures above belong to the Major Arcana. The remaining fifty-six minor cards comprise four suits numbered from Ace to 10 and including the four so-called court cards in each suit. The symbolism of four suits is related to four Jungian epistemic functions: thinking, sensing, feeling, and intuiting. The numerical growth from Ace to 10 represents the progressive mastery of a problematic situation, even when encountering a temporary defeat, as a lesson to be learned, that may be connoted by some numbered cards. The dynamics never stops: Tarot's imaginative narrative is full of stories about feeling happy or feeling sad; making plans or breaking promises; winning or losing; laying foundations for a marriage or getting out of an abusive relationship; falling in love or suffering from separation anxiety; starting a new venture or experiencing financial difficulties.

The ever expanding and varying multitude of experiential situations and events always presents new challenges, leading to the evolution of meanings expressed in the form of the semiotics of pictures and defying thereby Wittgenstein's pessimism. Like the illustrations to an adventure story, the images will have been imaginatively narrated by using all possible "keys" to decode the overall meaning and make the story of our own experience understood and cognized, thus bridging a dualistic gap between sensible and intelligible, between visible and invisible, between past and future.

The non-dual approach to the self other relation presupposes what Roger Penrose, non-incidentally, has defined in terms of a "contact with some sort of Platonic world" [50]. The core of Penrose's argument is that the physical world may be considered a projection of the Platonic world and the world of mind arises from part of the physical world, thus enabling one in this process to insightfully grasp and, respectively, understand some part of the Platonic world.

Because the Platonic world is inhabited by mathematical truths, but also due to the "common feeling that these mathematical constructions are products of our mentality", the mysterious, as Penrose was saying, dependence of the natural world on strict mathematical laws and the tri-relative relationship can be inscribed in the following-notably, triadic-diagram (Figure 12) [51]:

Figure 12. Three Worlds and three mysteries [50].

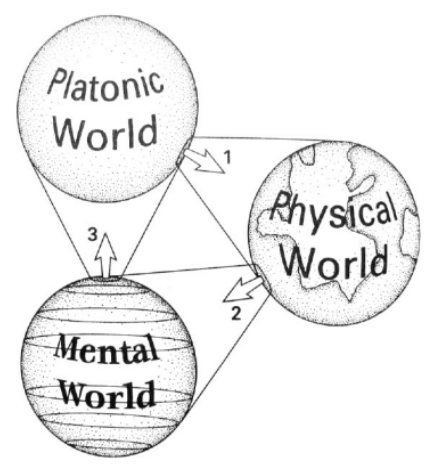


The relations stop being mysterious if we consider Penrose's diagram structured analogous to the Peircean triadic sign, hence uniting the three worlds in a manner akin to the triad in Figure 1. As a genuine sign, the Penrose's triad has to function reciprocally in accord with the logic of the included middle that would have enabled our insight into Platonic truths hence grasping the meanings of the (mathematical) concepts over following the logic of explicit computational rules. The approach of the computational universe as filled with information (or signs) supports the argument. An unorthodox quantum computation proceeds sub-consciously and pre-personally: the rules are not being followed explicitly, but implicitly, at the level of the virtual abstract machine that finds its embodiment in the semiotics of pictures.

The mathematical laws are expressed by a "part of Platonic world which encompasses our physical world” [50] and, by virtue of projecting itself onto the physical level, becomes "accessible by our mentality": we can anticipate the ideas that, by definition, "inhabit" the Platonic world of archetypes or ideal Forms. Or are they those very memes that by virtue of replication lay themselves down in the deep structures of the cultural unconscious?

Culture, according to Dennett's theory of memes, “influences the development of [the conscious] mind" [30]. What inhabits the Platonic world is not only the True but also the Good and the Beautiful, which appear to be, as Penrose says, "non-computable elements-for example, judgement, common sense, insight, aesthetic sensibility, compassion, morality" [50]-all the moral attributes of the psyche "encoded" in Major Arana. As the archetypal Platonic Ideas, they necessarily mediate between the world (physical world) and the intellect (mental world). Their participation in the evolutionary logic of the included middle enables the dynamic process of computing the apparently incomputable leading to anticipation of some future occurrences.

To repeat, the naturalistic approach to ethics does allow us to learn from experience and to evolve toward becoming able to do "the right thing at the right time" [11]. Our knowledge of Tarot language-or the machine code of the Mentalese-makes moral meanings computable, to a degree, as it allows us to decode them into words (verbal signs) describing dynamic patterns of thoughts, affects, desires, beliefs and even behaviors.

The rules of projective geometry implicit in the triadic structure establish the one-to-one correspondence (mapping, as we said earlier) like in a perspectival composition towards a vanishing point implying isomorphism between the archetypal ideas of the Platonic world and a dyad of the mental world together with the physical. We can conceptualize Penrose's triangle in terms of such a perspectival composition, but with a shifting frame of reference. When a "vanishing” point shifts into the mental world this leads to isomorphism between a generic mental representation and the other two worlds: the world of Ideas together with the world of our actions.

The level of Ideas-or moral meanings-must exceed (verbal) references because it encompasses our thinking (mental world) as coupled with our doing (physical world, the world of action). The Language of Thought by necessity exceeds its linguistic representations, and Jacques Lacan was correct when he said that unconscious too is structured as a language. And the language in question is the language of signs [52] that communicates itself along the memetic (semiotic) channels of information. And the ontological existence of the level at which such a symbolic language is "located" is implied by Penrose's triad. 
The Tarot layout as the projection [53] of the symbolic, informational, level of reality changes our understanding of nature. The natural world becomes what philosopher of mind David Chalmers, referring to Peirce's panpsychism, dubbed a strangely beautiful world. The beauty of this world consists in the fact that, above and over a simple physical and mental dyad, there is a triad as per Penrose's diagram, ensuring the very interaction or coupling of physical and mental worlds via its mapping and mediation through the world of Ideas, or memes, or archetypes.

If we literally step out of our Cartesian minds forever separated from the world and connect in practice with the world of our actions-as we do in case of Tarot readings-then we assume a position of what I call "radical objectivity", which is analogous to the implications of the so-called triangle (sic!) argument as shown below (Figure 13):

Figure 13. The Triangle Argument [from 54].

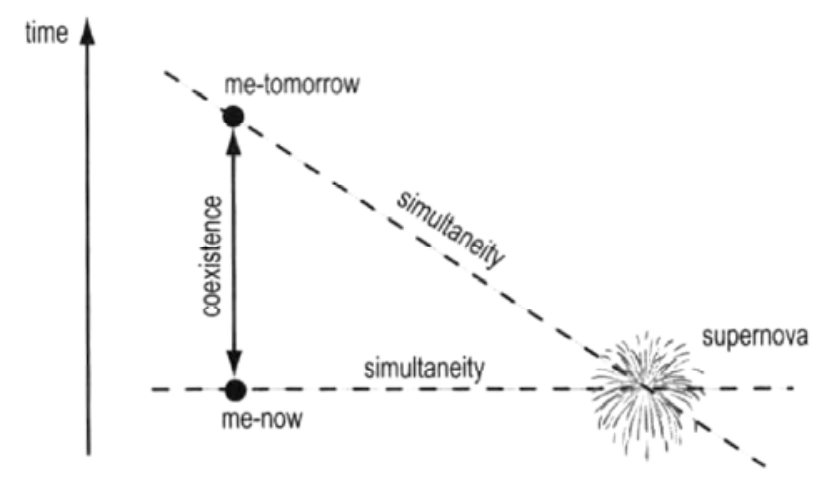

The imaginary "supernova" on this diagram is conceptually equivalent to the above-mentioned vanishing point in the perspectival composition that enables the projection leading to the simultaneity and co-existence of "me-now" with "me-tomorrow". The past-present-future co-exists as embedded in the same triadic, semiotic, structure (Figure 14):

Figure 14. A triadic relation.

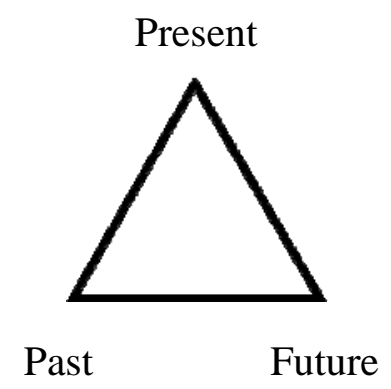

In agreement with Jantsch's "fine-structure of time" [19], a singular layout of images combines in itself all three aspects of time simultaneously, that enabling us to both forecast and backcast. The layout reflects on the possibility of anticipating the future by enabling the feeling of peculiar "gazing" into the possible future which may be described as "the options in further evolution" in the system's overall dynamics. The self-referential structure implies a circular causality vs. a mechanistic linear 
one. As David F. Griffin, a process philosopher, says, causation will have included a vertical dimension: "from the bottom up (projection) and then from the top down (reinjection) ...So-called precognition would really involve only the resonance of an event that is explicate now with an event that is later-from the viewpoint of the explicate order, which orders events sequentially to become explicated” [55].

Griffin refers here to explicate and implicate orders as posited by physicist David Bohm who also asserted the presence of the information in the universe and the significance of what he called active information. Lee Smolin in his quantum account of space and time, presents what he calls a week holographic principle that addresses cosmology in terms of events existing at the level below the level of ordinary human perception (cf. Lloyd's invisible information) and that can be made perceptible through "representations by which one set of events in the history of the universe receives information about other parts of the world" [56], that is, they can bee seen only in their projected format.

To repeat an analogy, a cinematic screen represents a 3D reality in only two dimensions: a loss in dimensions, or compactification, is thus implied. The screen metaphor is potent: it accords with the Tarot layout of pictures being spread on a surface as the means for meaning-making:

The area of a screen-indeed, the area of any surface in space-is really nothing but the capacity of that surface as a channel for information. So, according to the weak holographic principle space is nothing but a way of talking about all the different channels of communication that allow information to pass from observer to observer...In short, the holographic principle is the ultimate realization of the notion that the world is a network of relationships. These relationships are revealed by this new principle to involve nothing but information [56].

Recall the layout shown on Figure 2: not only does it represent a network of relationships connecting the disparate pictures in each position and creating an overall meaningful pattern, but it also includes the dimension of time: Tarot unorthodox epistemology is future-oriented and forward-looking. Tarot does extend the mind and does expand our consciousness encompassing the invisible-made-visible information in the guise of "me-tomorrow". As Erwin Laszlo pointed out, when the "patterns are perceived in a process, there is the possibility of extrapolation. Whatever the nature of the pattern, it provides a handle for grasping something about the way it will unfold in the future" [47].

Still, Laszlo does stop short of calling this vision a prediction, saying that the "nonequillibrium crystal ball does not foretell what will, only what is likely, to happen”. But the acquired expertise and knowledge of the pictorial language "spoken" by Tarot images necessarily increases the degree of "likelihood" and is crucial for "cracking” its code and understanding those implicit psychodynamic processes that long for interpretation and meaning making. During readings and because of the construction of imaginative narrative, the subject becomes "in-formed" and can literally change her perspective or her point of view in practice. Hence, the subject can become an object for itself, a self-referential object of its own signs in the guise of Tarot images when the formed complementary subject object relation enables the evolution of consciousness in practice and not only in theory. 


\section{Conclusion}

For a cognitive (computational) structure to fulfil its function it must be self-referential: a semiotic triangle (Figure 3) must operationally close on itself while leaving itself open to further evolution. The evolutionary process is characterized by a general law ultimately defined as the generalizing "tendency of all things to take habits" [6] that become the archetypal patterns in a continuous flow of semiosis constituting the process of the evolution of signs based on accumulating memes. This tendency is "self-generative”, and a genuine sign is ultimately self-referential.

We repeat that, as stated by Dennett [23], computational structures are self-referential hence capable of self-understanding. This is the ultimate value of Tarot: by means of self-reference and critical self-reflection we can achieve new understanding and even anticipation thus computing our own evolution as participating members in the computational universe. It is the self-reference that creates a non-linear evolutionary process by establishing a double-cycle in communication: from Stuart Kauffman's concept of the biosphere of nature to the semiosphere or memosphere of culture $[23,14]$ and back to the semiosphere as a real experiential phenomenon that expresses itself in a coded format of images; and vice versa from the cultural archetypes to the archetypes in nature projected into the layout in an array of pictures.

In the context of the computational and evolving universe, the archetypal ideas always have implicit content and meaning in the format of Lloyd's invisible information that becomes visible due to the mediating, interpretive, and anticipating function of Tarot readings. Every sign can always be further interpreted, the whole structure each time opening itself to a possibility of acquiring new meanings. The informational content (in a surprising agreement with Plato's old and so often considered irrelevant theory of knowledge) always already is, even if potentially or unconsciously.

Instead of being perpetual zombies behaving in the manner of automatons (what Peirce would have called the de-generate signs), the logic of which is reduced to the dyadic relations between the world of ideas and the physical world of blind and unconscious action, we can become capable of learning and evaluating our own evolution in the process of creative co-evolution. And what do we create? We create ourselves and our life-world just like the quantum universe as a whole [10] computes its own evolution; it self-organizes [19]. Peirce's semiosis is grounded in evolutionary philosophy but not because of its reliance on the Darwinian principle of natural selection. It is the greater realizations of meanings due to the chain of interpretants involved in a continuous semiotic communication that is a feature of organic evolution: "the man-sign acquires information and comes to mean more than he did before" [57].

The ethical question arises of how to treat the information that becomes available as a result of readings and implies, by virtue of its being a motivational force behind the transformation of habits, a possibility of producing new modes of action in the social world. The interpenetration of epistemology, ontology, and psychology by default leads to ethical connotations. The approach of a gentle action proposed by David Peat becomes a must. Back in 1992, Peat asserted that human actions qualified as gentle would have required an extraordinary quality of mind and perception based on love, respect and care. This type of action becomes especially important now, in our age of global conflicts, pluralistic values and the resulting breakdowns in communication and the alienation between "self" 
and "other". As this paper demonstrated, we can overcome the alienation by means of creating a connection (tilde $\sim$ ) embodied in the Tarot layout that establishes a complementary, even if precarious, self other relation.

The gentle action and caring attitude would respect an apparent "anomaly" of Tarot, which habitually rests on mantic signs being interpreted as an index of some supernatural forces. Considering this paper's perspective on computational universe and co-evolution, our increased awareness of the identical dynamic patterns acting in nature, culture, and the human mind should become both the necessary and sufficient condition for eliminating the prefix "super" from the "supernatural". The language of pictorial semiotics can take away the veil of ignorance. The real functioning of what appears to be a causal anomaly means that "the veil has apparently been pushed aside; we have been offered a glimpse of the deeper reality" [58].

Importantly-and despite the fact that the phenomenon of Tarot readings does support the idea of extreme innativism (on this I agree with Fodor)-the extension of the Mentalese to the universal level of proto-mentality demonstrates that it can and should be learned (that is, on this point I disagree with Fodor who insisted that we cannot learn it) via its mediation by physical marks. To conclude, we must take Fodor's project seriously as a step away from the habitual "linguistic turn" and a step forward towards developing both epistemology and ontology [59,60] of the necessary "semiotic turn" in our search for the pre-verbal "speech" and understanding the Mentalese. The symbolic level of the semiosphere (memosphere) presents itself to us in its projected form of the visible information encoded in the Tarot pictures that embody the otherwise hidden and invisible but potentially meaningful structures of collective experiences. The role of projective hypothesis in nature is strong: Henry Stapp posits “a certain mathematical 'projector' operator” [24] whose action appears to be direct (via projection) but that also causes indirect changes producing faster-than-light effects in the physical world.

This language of signs, symbols and images has been lost in the scientific pursuits of the modern epoch, during which we have "successfully" forgotten that once upon a time the whole Earth was of one language and of one speech, and the people were one (Genesis 11). To recollect this language presently is our ethical responsibility.

\section{References and Notes}

1. Dummett, M. The Game of Tarot: from Ferrara to Salt Lake City; Duckworth: London, UK, 1980.

2. Wittgenstein, L.; Pears, D.; McGuinness, B. Tractatus Logico-Philosophicus; Routledge: New York, NY, USA, 2001; p. 106.

3. Fodor, J.A. The Language of Thought; Harvard University Press: Cambridge, MA, USA, 1975; p. 214.

4. Semetsky, I. The end of a semiotic fallacy. SEMIOTICA 2000, 130, 283-300.

5. Nichols, S. Jung and Tarot : An Archetypal Journey; S. Weiser: New York, NY, USA, 1980.

6. Peirce, C.S. Collected Papers of Charles Sanders Peirce, 1860-1911; Hartshorne, C., Weiss, P., Eds.; Harvard University Press: Cambridge, MA, USA, 1931-1935.

7. Pauli, W. Writings on Physics and Philosophy; Springer-Verlag: Braunschweig, Germany,1994. 
8. Laszlo, E. The Interconnected Universe: Conceptual Foundations of Transdisciplinary Unified Theory; World Scientific: Singapore, 1995; p. 166.

9. Laszlo, E. Science and the Akashic Field: An Integral Theory of Everything, 2nd ed.; Inner Traditions: Rochester, VT, USA, 2007; p. 194.

10. Lloyd, S. Programming the Universe: A Quantum Computer Scientist Takes on the Cosmos, 1st ed.; Knopf: New York, NY, USA, 2006; p. 221.

11. Rockwell, W.T. Neither Brain nor Ghost: A Nondualist Alternative to the Mind-Brain Identity Theory; The MIT Press: Cambridge, MA, USA, 2007.

12. Sebeok, T. Communication. In A Sign is Just a Sign (Advances in Semiotics); Indiana University Press: Bloomington, IN, USA, 1991; pp. 23-35.

13. See some of my earlier publications in which I explored Tarot dynamics in terms of self-organization such as 1998, “On the Nature of Tarot”, section "Invited Opinions”, Frontier Perspectives, Center for Frontier Sciences: Temple University, PA, USA, Vol.7 (l), pp. 58-66; “The End of a Semiotic Fallacy”, SEMIOTICA 130-3/4, Berlin, New York, 2000: Mouton de Gruyter, pp.283-300; 2001, "Signs in action: Tarot as a self-organized system”, Cybernetics \& Human Knowing: a journal of second-order cybernetics, autopoesis and semiotics, special issue on Peirce and Spencer-Brown, vol. 8, no. 1-2, Imprint Academic, UK, pp. 111-132; 2001, "SelfOrganization in Tarot Semiotics”, in: Sign Processes in Complex Systems, Schmitz, W., Ed., Proceedings of the 7th International Congress of the IASS-AIS, ISBN:3-933592-21-6, Dresden: Thelem.

14. Lotman, Y. Universe of the Mind: A Semiotic Theory of Culture; Indiana University Press: Bloomington, IN, USA, 1990; p. 288.

15. Hoffmeyer, J. Signs of Meaning in the Universe; Indiana University Press: Bloomington \& Indianapolis, IN, USA, 1993.

16. Deely, J.N. Four Ages of Understanding: The First Postmodern Survey of Philosophy from Ancient Times to the Turn of the Twenty-First Century; University of Toronto Press: Toronto, Canada, 2001.

17. Jantsch, E. Design for Evolution: Self-Organization and Planning in the Life of Human Systems; G. Braziller: New York, NY, USA, 1975; p. 322.

18. Simon, H.A. Near decomposability and complexity: how a mind resides in a brain. In The Mind, the Brain, and Complex Adaptive Systems. Proceedings of the Santa Fe Institute in the Sciences of Complexity, Morowitz, H.J., Singer, J.L., Eds.; Addison-Wesley: Reading, MA, USA, 1995; Volume XXII, pp 25-43.

19. Jantsch, E. The Self-Organizing Universe: Scientific and Human Implications of the Emerging Paradigm of Evolution, 1st ed.; Pergamon Press: Oxford ; New York, NY, USA, 1980; p. 343.

20. Kelso, J.A.S.; Engstrøm, D.A. The Complementary Nature; MIT: Cambridge, MA, USA, 2006.

21. Nicolescu, B. Towards transdisciplinary education and learning. In Science and Religion: Global Perspectives; Metanexus Institute: Philadelphia, PA, USA, 2005.

22. Kauffman, L.H. Virtual logic. Syst. Res. 1996, 13, 293-310.

23. Dennett, D.C. Brainstorms: Philosophical Essays on Mind and Psychology, 1st ed.; Penguin: London, UK, 1997; p. 353. 
24. Stapp, H. Mindful Universe: Quantum Mechanics and the Participating Observer; SpringerVerlag: Berlin, Germany, 2007.

25. Jantsch, E. Unifying principles of evolution. In The Evolutionary Vision: Toward a Unifying Paradigm of Physical, Biological, and Sociocultural evolution, Jantsch, E., Ed.; Westview Press: Boulder, CO, USA, 1981; pp. 83-116.

26. Semetsky, I. Learning by abduction: a geometrical interpretation. SEMIOTICA 2005, 157, 199-212.

27. Guiraud, P. Semiology; Routledge \& K. Paul: London, UK, 1975; p. 106.

28. Dennett, D.C. Kinds of Mind: Toward an Understanding of Consciousness. Basic Books: New York, NY, USA, 1996.

29. Heschl, A. The Intelligent Genome; Springer-Verlag: Berlin, Germany, 2002.

30. Popp, J.A. Evolution's First Philosopher: John Dewey and the Continuity of Nature; State University of New York Press: Albany, NY, USA, 2007.

31. Markoš, A. In the quest for novelty: Kauffman’s biosphere and Lotman’s semiosphere. Sign Syst. Stud. 2004, 1-2, 309-327.

32. Nöth, W. Handbook of Semiotics; Indiana University Press: Bloomington, IN, USA, 1995.

33. Semetsky, I. Integrating tarot readings into counselling and psychotherapy. Spirituality Health Int. 2006, 6, 81-94.

34. Brier, S. Biosemiotics and the foundation of cybersemiotics. Reconceptualizing the insights of Ethology, second order cybernetics and Peirce's semiotics in biosemiotics to create a nonCartesian information science. Semiotica 1999, 127, 169-198.

35. Brier, S. Cybersemiotics: Why Information is Not Enough; University of Toronto Press: Toronto, Canada, 2008.

36. Thom, R. From the icon to the symbol. In SEMIOTICS: An Introductory Anthology, Innis, R.E., Ed.; Indiana University Press: Bloomington, IN, USA, 1885.

37. Rosen, R. Life Itself: A Comprehensive Inquiry into the Nature, Origin, and Fabrication of Life; Columbia University Press: New York, NY, USA, 1991.

38. Samuels, A. Jung and the Post-Jungians; Routledge \& K. Paul: London, UK, 1985.

39. See Atom and Archetype: The Pauli/Jung Letters 1932-1958. Edited by C.A. Meier, with a preface by Beverley Zabriskie (2001, Princeton University Press). This particular letter is designated in the book as 56P, pp. 81-83. See also my 2006 article "The language of signs: Semiosis and the memories of the future", SOPHIA: International Journal for philosophy of religion, metaphysical theology and ethics, Volume 45, No.1, pp. 95-116.

40. Jackendoff, R. Language in the ecology of mind. In The Routledge Companion to Semiotics and Linguistics, Cobley, P., Ed.; Routledge: London, UK, 2001; pp. 52-65.

41. The illustrations are from Rider-Waite Tarot Deck, known also as the Rider Tarot and the Waite Tarot. Reproduced by permission of US Games Systems Inc., Stamford, CT 06902, USA. Copyright 1971 by US Games Systems, Inc. Further reproduction prohibited.

42. I suggested a possible solution to this paradox in a number of my publications, the most recent "Resolving the Socratic Paradox: A semiotic approach", in K. Boudouris and M. Knezevic (Eds.), Paideia: Education in the Global Era, Vol. I, Ionia Publications, Athens 2008, pp. 207-221; and 
"Deleuze as a philosopher of education: Affective knowledge/Effective learning" in The European Legacy: Toward New Paradigms, Volume 14, Issue 4, (July 2009), pp. 443-456.

43. The meaning of "The Magician" has been analysed in detail in a number of my publications starting from the 2003 article "The Magician's autopoietic action, or Eros contained and uncontained”, Trickster's Way, Volume 2, Issue 3 http://www.trinity.edu/org/tricksters/TrixWay. The developed version titled "The transversal communication: reconciling science and magic" appeared in Cybernetics \& Human Knowing, 2008, Volume 15, No. 2, pp. 33-48. The new expanded 10000-word version titled "The Magician in the World: becoming, creativity, and transversal communication” is published in June 2009 in ZYGON: Journal of Religion \& Science, Volume 4, No. 2, pp. 323-346.

44. Merrell, F. Peirce, Signs, and Meaning; University of Toronto Press: Toronto, Canada, 1997; p. 384.

45. Barrow, J.D. The Book of Nothing; Vintage Books: New York, NY, USA, 2000.

46. Rucker, R. Infinity and the Mind: The Science and Philosophy of the Infinite; Birkhauser: Basel, Switzerland, 1982.

47. Laszlo, E. The Age of Bifurcation: Understanding the Changing World; Gordon and Breach: Philadelphia, PA, USA, 1991; p. 126.

48. Semetsky, I. Semanalyisis in the age of abjection. Appl. Semiotic. 2006, 17, 24-45.

49. This image is from the deck called The Lovers' Tarot, by Jane Lyle; Illustration Copyright (C) Oliver Burston 1982. The pack is published by Connections (January 2000) in the UK and St. Martin's Press in the US.

50. Penrose, R.; Shimony, A.; Cartwright, N.; Hawking, S. The Large, the Small, and the Human Mind; Cambridge University Press: Cambridge, UK, 1997.

51. I addressed this argument (reproduced here with minor changes) in my paper "From design to self-organization, or: a proper structure for a proper function" published in 2005 in AXIOMATHES: An International Journal in Ontology and Cognitive Systems, Vol. 15, No. 4, pp. 575-597.

52. Semetsky, I. The language of signs: Semiosis and the memories of the future. SOPHIA: Int. J. Phil. Relig Metaphysical Theol. Ethics 2006, 45, 95-116.

53. Semetsky, I. Tarot as a projective technique. Spirituality Health Int. 2006, 7, 187-197.

54. Kennedy, J.B. Space, Time and Einstein: An Introduction; Acumen Publishing Limited: Chesham, UK, 2003.

55. Griffin, D.R. Physics and the Ultimate Significance of Time: Bohm, Prigogine, and Process Philosophy; State University of New York Press: Albany, NY, USA, 1986; p. 322.

56. Smolin, L. Three Roads to Quantum Gravity; Basic Books: New York, NY, USA, 2001.

57. Peirce, C. S., Philosophical writings of Peirce. Dover Publications: New York, 1955.

58. Stapp, H. Mind, Matter and Quantum Mechanics; Springer-Verlag: Berlin, Germany, 1993.

59. Semetsky, I. The Magician in the World: becoming, creativity, and transversal communication. Zygon: J. Relig. Sci. 2009, 4, 323-346. 
60. Semetsky, I. Virtual ontology/Real experiences. Proceedings of the Cosmos, Nature, Culture: A Transdisciplinary Conference, Metanexus Institute, Phoenix, AZ, USA, 2009.

(C) 2010 by the authors; licensee Molecular Diversity Preservation International, Basel, Switzerland. This article is an open-access article distributed under the terms and conditions of the Creative Commons Attribution license (http://creativecommons.org/licenses/by/3.0/). 